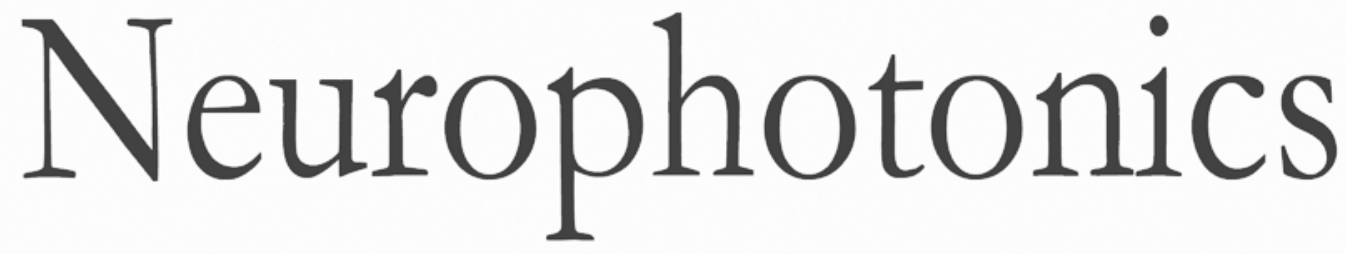

\title{
Review on the role of AMPA receptor nano-organization and dynamic in the properties of synaptic transmission
}

\author{
Benjamin Compans \\ Daniel Choquet \\ Eric Hosy
}




\title{
Review on the role of AMPA receptor nano-organization and dynamic in the properties of synaptic transmission
}

\author{
Benjamin Compans, ${ }^{\mathrm{a}, \mathrm{b}}$ Daniel Choquet, ${ }^{\mathrm{a}, \mathrm{b}, \mathrm{c}, *}$ and Eric Hosy ${ }^{\mathrm{a}, \mathrm{b}}$ \\ aUniversity of Bordeaux, Interdisciplinary Institute for Neuroscience, UMR 5297, Bordeaux F-33000, France \\ Interdisciplinary Institute for Neuroscience, CNRS, UMR 5297, Bordeaux F-33000, France \\ 'University of Bordeaux, Bordeaux Imaging Center, UMS 3420 CNRS, US4 INSERM, France
}

\begin{abstract}
Receptor trafficking and its regulation have appeared in the last two decades to be a major controller of basal synaptic transmission and its activity-dependent plasticity. More recently, considerable advances in super-resolution microscopy have begun deciphering the subdiffraction organization of synaptic elements and their functional roles. In particular, the dynamic nanoscale organization of neurotransmitter receptors in the postsynaptic membrane has recently been suggested to play a major role in various aspects of synapstic function. We here review the recent advances in our understanding of alpha-amino-3-hydroxy-5-méthyl-4-isoxazolepropionic acid subtype glutamate receptors subsynaptic organization and their role in short- and long-term synaptic plasticity. ๑ The Authors. Published by SPIE under a Creative Commons Attribution 3.0 Unported License. Distribution or reproduction of this work in whole or in part requires full attribution of the original publication, including its DOI. [DOI: 10.1117/1.NPh.3.4.041811]
\end{abstract}

Keywords: receptor trafficking; synaptic plasticity; super-resolution microscopy.

Paper 16041SSVRR received Jun. 21, 2016; accepted for publication Oct. 19, 2016; published online Nov. $15,2016$.

\section{Introduction}

The fundamental building block of neuron-to-neuron communication is the synapse, a micrometer size organelle, where the membranes of two cells come in close apposition to favor information transfer. Our deep understanding of this structure, named for the first time in 1897 by Foster and Sherrington, has evolved in parallel with the development of new technologies. Most of the main conceptual advances in our understanding of synaptic organization and function have originated from new imaging developments. Based on the new silver staining developed by Camillo Golgi, Cajal ${ }^{1}$ demonstrated that nerve cells are not continuous but contiguous, invalidating the cable theory of the nervous system. At the same time, he introduced the notion that a synapse is composed of three independent compartments: the presynapse, the postsynapse, and the space between them: the synaptic cleft. This organization remained hypothetical until the first precise image of a synapse was obtained in parallel in the 1950s by two laboratories using electron microscopy. ${ }^{2,3}$ The first image of a synapse revealed an asymmetric organization, with one compartment enriched in $\sim 50 \mathrm{~nm}$ sized vesicle., ${ }^{2,4,5}$ This discovery and the demonstration one year later that these vesicles contained neurotransmitters, ${ }^{5}$ coupled to Katz's electrophysiological recordings of unitary postsynaptic voltage changes, established most of the basis for our current knowledge of the mechanisms of synaptic transmission. ${ }^{6,7}$ The presynapse releases a "quantum" of neurotransmitters in the synaptic cleft due to discrete vesicle fusion, triggering a reproducible postsynaptic current. Despite the large number of newly available techniques, our present vision of the synapse is not very different from the one described by Palay, even though the invention of the patch-clamp technique offered a more robust way to

*Address all correspondence to: Daniel Choquet, E-mail: daniel.choquet@ u-bordeaux.fr measure synaptic currents ${ }^{8}$ and the revolution in genomics and proteomics allowed to allocate proteins, their interactions, and structures, into the various synaptic compartments. From the cloning of the first glutamate receptor in $1994^{9}$ and the identification of PSD-95 as the main scaffold element of the postsynaptic density, ${ }^{10-12}$ to the extensive proteomic characterization of synaptic elements, ${ }^{13-16}$ it is probably safe to say that by now, most protein constituents of the synapse have been identified. However, as detailed below, we still do not fully understand how synapses work and many shadow zones remain.

An important misconception in shaping our original understanding of synaptic transmission was the omission of dynamic regulation at various levels. Indeed, since 1973 and the discovery of the concept of synaptic plasticity by Bliss and Lomo, new dynamic levels of regulation of synaptic transmission have regularly been identified. From this moment, synaptic transmission is accepted as a dynamic mechanism, which can be modified through plastic events on both short and long terms to adapt the synaptic transmission to various types of received inputs. ${ }^{17-20}$

The expansion of neuroscience research during the 1990s led to an intense debate over the role of both the pre- and the postsynapse in those plastic events. Short-term plasticity has been usually attributed to presynaptic modifications. Briefly, when action potentials arrive in the $1-$ to $100-\mathrm{Hz}$ range, calcium levels accumulate over time in the presynaptic terminal, leading to a time-dependent increase in the release probability, which is responsible for short-term paired-pulse facilitation. ${ }^{21}$ This dogma is still valid in spite of the identification of some postsynaptic components in the regulation of short-term synaptic depression, such as alpha-amino-3-hydroxy-5-méthyl-4-isoxazolepropionic acid (AMPA) receptor (AMPAR) desensitization and more recently AMPAR lateral diffusion (see Sec. 2.1).

Concerning long-term plasticity, the debate has been more pronounced. The main evidence suggesting a presynaptic mechanism came from the observation that the synaptic failure 
rate decreases following the induction of long-term potentiation (LTP) ${ }^{22-24}$ But other studies suggest that postsynaptic modifications, such as AMPAR over-accumulation, were sufficient to induce LTP. ${ }^{25-28}$ Various recent studies demonstrate that the reality lies in-between. Postsynaptically, changes in the number and composition of AMPAR complexes have been observed by uncaging and fluorescence imaging experiments. Moreover, some synapses are able to unsilence following potentiation protocols by accumulating AMPAR. ${ }^{29-31}$ On the other hand, retrograde signaling via endocannabinoids indicates that the presynapse is also affected by long-term plasticity and, until now, the existence of a possible increase in glutamate content inside vesicles, or the change of release probability has not been ruled out. $^{32,33}$

This review paper focuses mainly on postsynaptic organization and modifications, but it is important to constantly keep in mind that pre- and postsynapses are intrinsically connected and coregulated. We will focus on changes that occur on the postsynaptic side of the synapse, which indeed are now recognized as playing a central role in plasticity at many synapses, including the Schaeffer collaterals and CA1 pyramidal cells of the hippocampus, arguably the best studied synapse in terms of plasticity phenomenon.

Modifications in postsynaptic properties have been proposed early to account for plasticity of synaptic transmission. ${ }^{34-36}$ These modifications have been attributed both to the changes in glutamatergic receptor properties ${ }^{26,37-39}$ and the modification in AMPAR numbers at the postsynapse. ${ }^{30,35,40,41}$ The changes in AMPAR number have been initially attributed solely to endocytic and exocytic processes. ${ }^{42-46}$ It has been demonstrated that exocytosis of AMPAR is essential for induction of LTP. ${ }^{44}$ But an important remaining question was how do AMPARs travel from the exocytosed vesicle to the synapse? The first use of singleparticle tracking, the ancestor of super-resolution microscopy, revealed that AMPAR can diffuse at the plasma-membrane (as all transmembrane proteins, and in particular all neurotransmitter receptors) and exchange between synaptic and extrasynaptic sites. ${ }^{47-49}$ The application of the revolutionary singleparticle and single-molecule-tracking approaches has granted access to understanding the behavior of single proteins. After a series of first steps based on imaging latex beads, then organic dyes and semiconductor quantum dots, the last decade has seen a large development of super-resolution imaging techniques largely based on massively increasing the throughput of single-molecule detection assays, offering a new vision of synapse organization.

\section{New Vision of the Synapse}

\subsection{Nonsaturation of Postsynaptic AMPARs by Glutamate Release}

The conceptualization of the synapse as being composed of a presynaptic compartment dedicated to calcium-dependent neurotransmitter release and a postsynaptic compartment harboring a stable number of receptors has long been sufficient to define a functional model of synaptic transmission. Within such a framework, long-term plasticity is explained by presynaptic modification of release probability and potential changes in the glutamate content per vesicle, and by postsynaptic increases or decreases in the total amount of AMPAR inside the PSD. Our view of the number of AMPAR present in a given PSD has evolved importantly over the years. One of the initial paper, based on electron microscopy, described a "sharp decrease of receptor density at the edge of the membrane specialization (the PSD), which demonstrates that at a given level of glutamate only a well-defined number of receptors can be activated." ${ }^{, 50}$ Even if glutamate diffuses out of the cleft, a much lower density of receptors will be reached, probably contributing little to the synaptic current. Then, improvement in fluorescence microscopy and electron microscopy labeling and glutamate uncaging started to better estimate the number of AMPAR inside the synapse, with an amount of around 100 receptors per synapse. ${ }^{51-53}$ A paradox appeared when the number of AMPAR per PSD was compared to the effective amplitude of miniature currents, which reports a lower amplitude than expected even by taking into account the low affinity of AMPAR for glutamate.

The first answer to this paradox has been brought by the Richard Tsien Laboratory, when they demonstrated that a single glutamate vesicle release into the synaptic cleft was not able to saturate all postsynaptic AMPARs. ${ }^{54}$ This work has then been confirmed by other laboratories, even if the real saturation level of AMPAR inside the synapse during endogenous activity is still not perfectly defined. ${ }^{55-59}$ Indeed, experimental studies of glutamate diffusion into the synaptic cleft suggest that under the release site, glutamate can reach a concentration of around 1 to $5 \mathrm{mM}$ within a couple $100 \mu$ s following vesicle release. ${ }^{57,58,60-62}$ Computing and modeling, based largely on Monte Carlo simulations, allowed to estimate the width of the synaptic area, where glutamate concentration is sufficient to activate AMPAR. Due to the strong cooperativity of AMPAR activation and the rapid dissipation of glutamate, AMPAR seems to be activated only onto an area of around 100 to $150 \mathrm{~nm}$ full width at half maximum (FWHM) in front of the release site..$^{5,56,59,62,63}$ These conclusions partly change our conception of what could be the synaptic quantum of response. Indeed, initially a quantum was considered as the number of glutamate molecules per vesicle. Models now show that the amplitude of synaptic responses depends not only on the presynaptic quantum but also on the clustering level of AMPARs and their position with respect to the release site (Fig. 1). ${ }^{55,59,64,65}$

\subsection{Lateral Diffusion of AMPARs as a Mechanism to Control AMPAR Density at the Synapse}

Although the concept of a fluid mosaic membrane has been proposed since 1972 by Singer and Nicholson, ${ }^{66}$ and that the application of the FRAP technique has demonstrated a rapid exchange via Brownian lateral diffusion of the various membrane constituents, ${ }^{67,68}$ it is only since the early 2000 s, with the improvement of single-particle tracking techniques, that lateral diffusion has started to be considered as a nonnegligible physiological parameter, particularly in neuronal cells. Precursor studies were performed by Mu Ming Poo's Lab on the acetylcholine receptor, showing its diffusion in the extrasynaptic membrane of muscle cells and introducing the diffusion trap model ${ }^{68-70} \mathrm{~A}$ few years later, many laboratories, including Sheetz's to study adhesion molecules and biomechanical forces and Kusumi's to understand diffusion properties of membrane proteins and lipids, have used and improved single-particle tracking techniques. ${ }^{71-73}$ In 2001, for the first time, our group together with Antoine Triller applied single-particle tracking techniques on neurons to reveal and analyze the properties of the mobility of an inhibitory neurotransmitter receptor. ${ }^{74}$

One year later, we published the characterization of AMPAR surface mobility. ${ }^{47}$ The use of single-particle tracking drastically 
(a) Glutamate saturates AMPA receptors

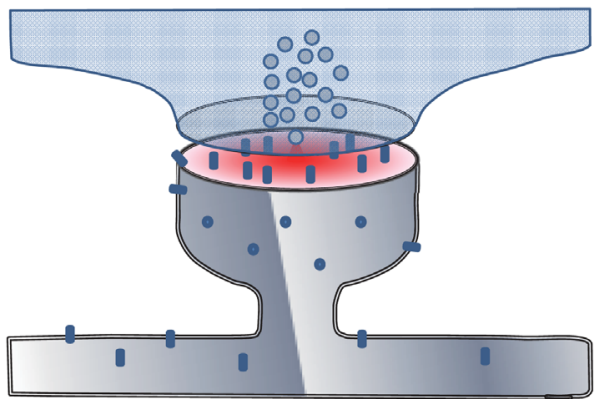

(b)
Uneven organization Random release
Local activation of AMPAR

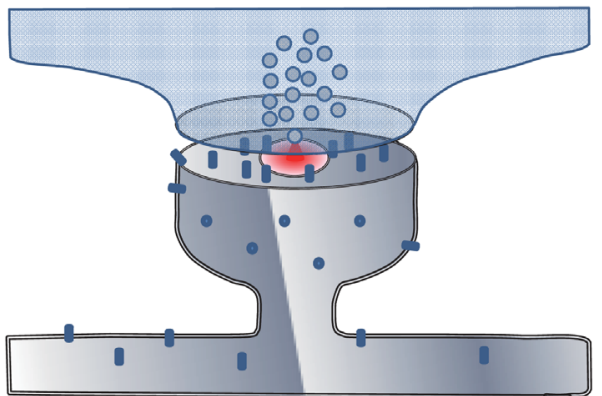

Uneven organization and pre-post alignment

(c)
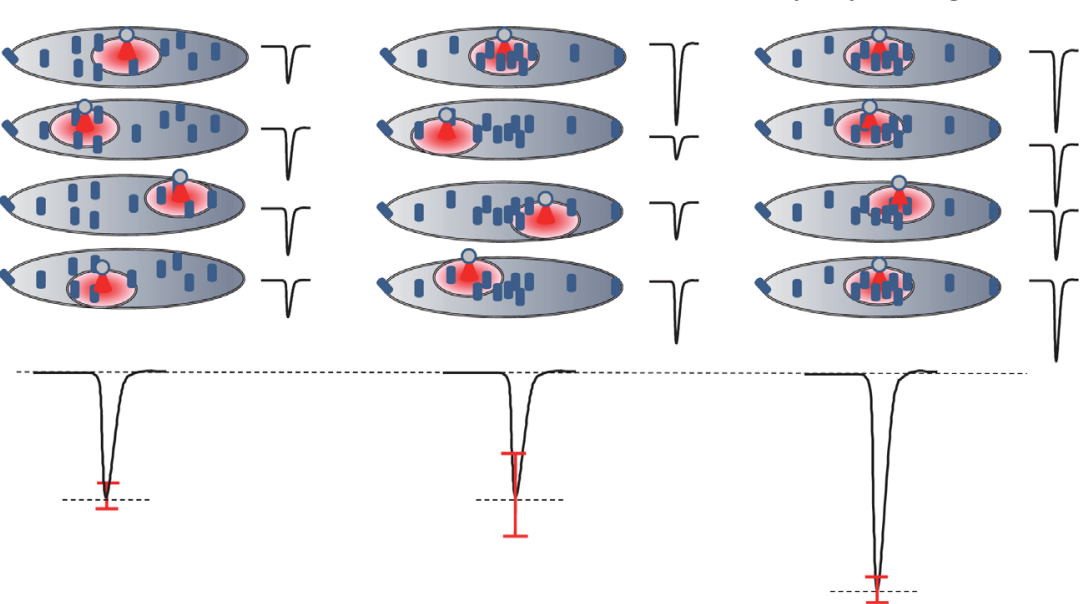

Fig. 1 Toward a new vision of the synapse. (a) Scheme of synapse, the area where AMPAR can be activated by glutamate after release of a presynaptic vesicle is represented in red. Previously, synaptic receptor in the synapse was thought to be saturated, in 1920s experiments from several laboratories demonstrated that AMPAR are likely activated only on a 100- to 150-nm diameter area due to their low affinity. ${ }^{55,56,59,62}$ (b) Effect of AMPAR organization and release site localization on the variability of AMPAR responses. Following the discovery of the nonsaturation of synaptic AMPAR, modeling studies identified three hypothesis represented here. From left to right: even organization of AMPAR and random release, clustered AMPAR and random release, clustered organization and release in front of the cluster. The corresponding average and variability of miniature EPSC in function of AMPAR organization are represented in (c).

changed our vision of AMPAR dynamic and organization inside synapses. The dogma that neurotransmitter receptors were immobile at synapses, their number in the PSD being affected only by endo- and exocytosis, was proven wrong. Indeed, various experiments revealed that AMPARs constantly alternate between fast Brownian diffusion and confined motion. ${ }^{47,49}$ Each receptor may adopt successively both of these behaviors, and activity regulates the time spent in one or the other diffusive state. $^{49,75-78}$ Importantly, these experiments revealed the presence of specific and saturable binding sites for AMPAR inside the synapse.

The following years in the field have been dedicated to identify which molecular mechanisms are responsible for the AMPAR trapping at synapses. Unraveling the nature of the traps was intimately linked to the initial progress in genome sequencing and decoding and then the improvement in high throughput and sensitive proteomic technique. ${ }^{79-82}$ For example, Letts et al. ${ }^{83}$ cloned gamma2, a protein belonging to the calcium channel family that when mutated triggered hereditary epilepsy in mice. Two years later, gamma2 (also named stargazin) has been identified as the first AMPAR regulatory protein, implicated in both their cellular traffic to the membrane, the regulation of their electrophysiological properties and responsible for their synaptic trapping. ${ }^{84,85}$ These studies demonstrated that AMPARs do not travel alone, but they are part of a macromolecular complex composed of many different auxiliary proteins. The composition of these complexes is highly dynamic and varies across different brain regions and during neuronal activity. ${ }^{86}$ So far, the AMPAR complex proteome is composed of $>30$ different proteins, mainly transmembrane ones. It includes the receptor core, formed by tetramers of the pore forming GluA1-4 subunits ${ }^{9,87}$ and of various associated proteins belonging mainly to three families of membrane proteins: the transmembrane AMPA regulatory proteins (TARPs $\gamma-2, \gamma-3, \gamma-4, \gamma-7$, and $\gamma-8,{ }^{88}$ ), the cornichon, (CNIH2 and CNIH3 $3{ }^{81,89}$ ), and the shisa family (Shisa9/ CKAMP44 and Shisa6, ${ }^{82,90,91}$ ) [Fig. 2(a)]. The precise role of each auxiliary subunit is not well established, even if many studies using knock-out mice or protein mutations have tried to clarify the impact of some AMPAR associated proteins on synaptic function both at basal state and during plastic events. 
(a) TARP family

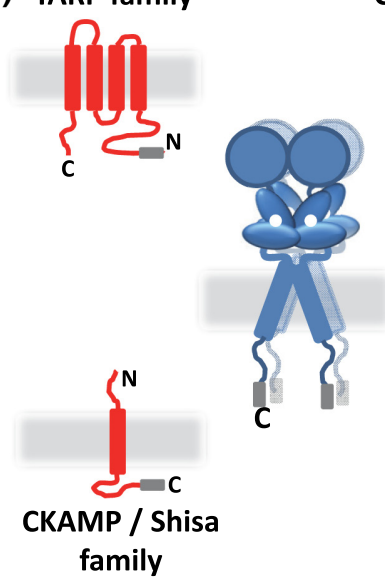

Cornichon family

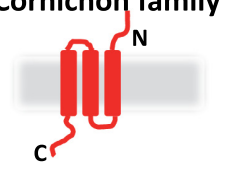

GSG1L

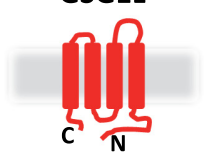

(b)

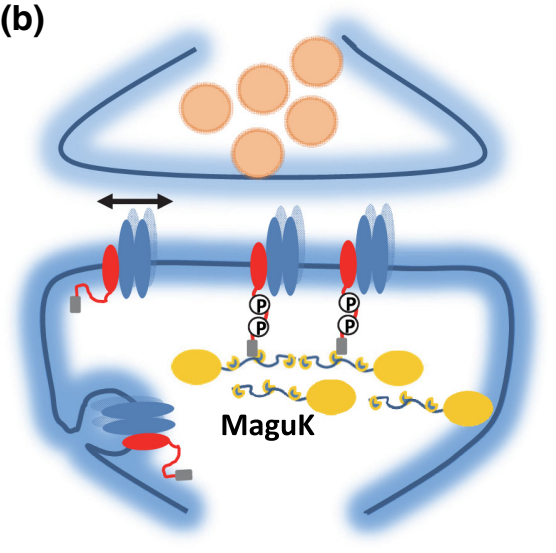

Fig. 2 Scheme of the molecular organization of AMPAR content. (a) Representation of the AMPAR (as a tetrameric structure) and of the various identified auxiliary proteins structure. (b) Schematic representation of an example of molecular AMPAR complex stabilization inside synapse. The phosphorylation of the cytoplasmic tail of stargazin favors its orientation to the cytosol, increasing its interactions with scaffolding proteins, and so immobilizing the AMPAR complex. ${ }^{92,93,94}$ Nonphosphorylated complexes present a higher lateral surface mobility.

The most studied auxiliary proteins belong to the family of the TARPs, which include stargazin (TARP $\gamma$-2), the canonical member of this family. Stargazin is important for the trapping of AMPARs inside the synapse and more particularly to the MAGUK proteins present inside the PSD (such as PSD95 ${ }^{84,92}$ ). The loss of interaction between the TARP and the scaffold, as shown using a c-terminus truncation mutant of stargazin that cannot bind PSD95 (delta-C mutant), impairs AMPAR accumulation at synapses, decreasing the amplitude of the synaptic response. ${ }^{95}$ Single-particle tracking video microscopy demonstrated that the dynamic interaction between stargazin and PSD-95 regulates the exchange of AMPARs by lateral diffusion between extrasynaptic and synaptic compartments. ${ }^{95}$ Those exchanges are controlled mostly by the phosphorylation state of the TARP ${ }^{92,93,96}$ [Fig. 2(b)]. The disruption of this interaction using competing divalent ligands reduces AMPAR synaptic function and decreases the trapping of AMPAR at synapses. $^{97}$ Interestingly, competing for the TARP-PSD95 interaction could suppress only half of the synaptic responses, suggesting that other interactions might be at play to stabilize AMPAR at synapses.

Little is known about the role of other TARPs on AMPAR lateral diffusion and immobilization at the PSD. TARP $\gamma$-7, mainly expressed in the cerebellum, seems to be also involved in the regulation of AMPAR anchoring inside the synapse, ${ }^{98,99}$ and TARP $\gamma-8$, mainly expressed in the hippocampus and in the cortex, seems to control AMPAR number at the plasma membrane and extrasynaptic localization, ${ }^{100}$ even if its role in anchoring to PSD-95 is still controversial. ${ }^{100,101}$

The literature is less abundant concerning the auxiliary proteins that do not belong to the TARP family, and for the moment, a clear vision of their physiological and molecular role is still lacking. The cornichon protein seems to be able to form a tripartite interaction with AMPAR and TARP. ${ }^{102}$ This interaction could stabilize AMPAR/TARP complex and act on AMPAR gating properties. ${ }^{89}$ Initially, the shisa family members had been identified as a regulator of the biophysical properties of AMPARs ${ }^{82,90,91,103}$ but recently, Klaassen et al..$^{91}$ demonstrated that they also play a role in anchoring AMPAR. All those studies pointed to the existence of a tight coupling between the regulation of AMPAR gating properties and their diffusion/trapping behavior. Despite extensive research on the role of the different auxiliary protein on AMPAR properties, heavy work is still needed to determine the contribution of the AMPAR complex composition variability into the multiplicity of synaptic response properties observed in the different central nervous system areas.

Even if the precise role of each AMPAR auxiliary subunit is not clear, previous studies have shown that they play a crucial role in both the lateral diffusion and the synaptic organization of AMPAR, thus regulating the synaptic transmission efficiency. Most of these experiments used quantum dot or FRAP experiments, limiting the access to a high number of individual molecule properties. The emergence of new high-density live super-resolution techniques with higher throughput will now allow better characterization of the role of each auxiliary protein in AMPAR organization and diffusion properties.

\subsection{Postsynaptic Nano-Organization}

As mentioned above, studies in the early 2000s questioned the existence of a putative sub-PSD organization of postsynaptic proteins. $^{54,56,59}$ Unfortunately, optical microscopy is limited by diffraction to $300 \mathrm{~nm}$, rendering it impossible to decipher AMPAR organization with a precision higher than the PSD size. First attempts at describing this organization have been performed using single-particle tracking with quantum dots. In these conditions, random second to minute time scale immobilization of AMPAR in the PSD was reported, revealing a potential local subsynaptic organization. ${ }^{76}$ But it is only the recent application of the new super-resolution microscopy techniques on AMPAR that succeeded to reveal the AMPAR nano-organization inside synapses. ${ }^{64,65,104-106}$

In the last decade, new microscopy techniques have been developed to bypass the diffraction limit, such as structured illumination microscopy, stimulated emission depletion (STED), and single-molecule localization microscopy, including photoactivated localization microscopy (PALM), universal point 
(a) Synaptic AMPAR nano-organization

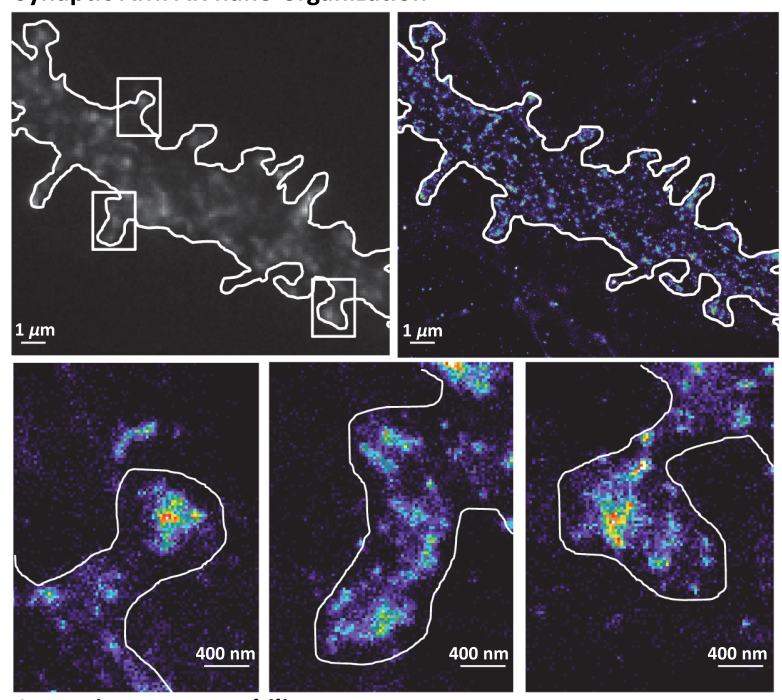

(b)
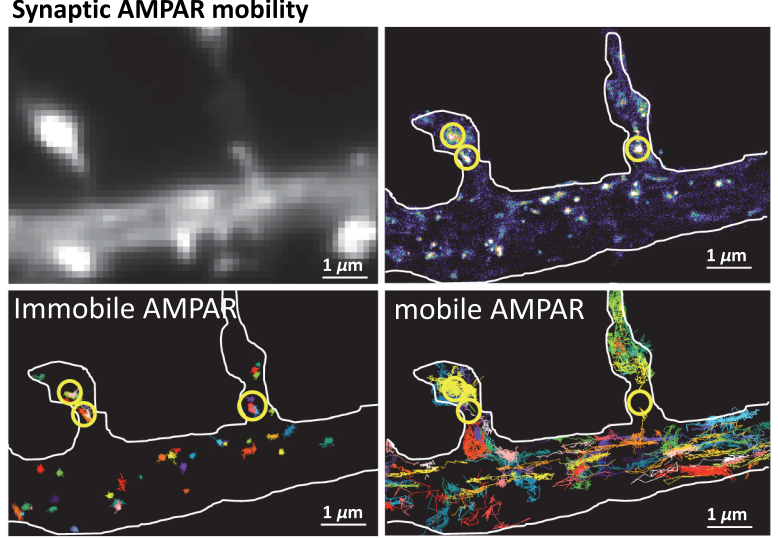

Fig. 3 Example of AMPAR nano-organization and lateral mobility. (a) Image of conventional fluorescence images and high-resolution d-STORM of AMPAR organization on a dendrite (upper part), with zoom on three synapses, where clusters can be easily distinguished (lower part). (b) Image of conventional fluorescence images and highresolution u-PAINT of AMPAR lateral mobility on a dendrite (upper part). In the lower part are represented individual AMPAR trajectories of immobile receptors (left panel), which are mainly presented inside nanoclusters (yellow circle), and mobile receptors (right panel), which are enriched out of the nanoclusters.

accumulation in nanoscale topography (u-PAINT), and stochastic optical reconstruction microscopy (STORM). ${ }^{107-115}$ These techniques allow observation of biological samples with 10to $100-\mathrm{nm}$ spatial resolution. The improvement in labeling techniques, fluorescent probes, and optical parameters has led to major improvements in this field and opened the possibility today to perform multicolor three-dimensional (3-D) image acquisitions at tens of nanometer resolution, ${ }^{116-120}$ in tissue, ${ }^{121-124}$ or even in vivo. ${ }^{125-128}$ This improvement in superresolution imaging also led to the development of high-density single-particle tracking at the nanoscale. The most used approach is arguably sptPALM,${ }^{114}$ which allows tracking target proteins genetically fused with photoswitchable fluorescent proteins. More recently, the development of u-PAINT allowed for the first time to track a high density of endogenous membrane proteins and to build super-resolved images of native proteins in real time by stochastic labeling. ${ }^{110}$

The emergence of those super-resolution imaging techniques and their application in neuroscience allows a better understanding of the dynamic distribution of synaptic proteins at the nanoscale. In 2010, for the first time, STORM on fixed olfactory bulb slices was performed to map the organization of various pre- and postsynaptic scaffolding proteins. ${ }^{122} \mathrm{~A}$ few years later, three papers using different complementary super-resolution techniques were published and tackled the question of the nano-organization of postsynaptic AMPARs and PSD-95. ${ }^{64,65,105}$

Using a combination of super-resolution techniques, on fixed or living hippocampal cultured neurons, Nair et al. focused on AMPAR's dynamic nano-organization. Using u-PAINT and sptPALM, they tracked AMPARs at high density and showed for the first time the presence at synapses of AMPARs nanodomains. They observed that AMPARs are immobilized in fixed hotspots and are mobile between those. Super-resolution imaging on fixed cells (u-PAINT, PALM, dSTORM, and STED), as well as electron microscopy, confirmed the presence of one to three $80 \mathrm{~nm}$ clusters per synapse containing 20 to 25 receptors each (Fig. 3). Those AMPAR nanodomains can be stable for tens of minutes at the synapse as shown by time lapse sptPALM. ${ }^{65}$ On the other hand, MacGillavry et al. ${ }^{64}$ studied the dynamic organization of PSD-95-mEOS by PALM and sptPALM and showed the presence of one 80-nm clusters per synapse. Fukata et al. ${ }^{105}$ via an elegant approach, observed $\sim 150$-nm cluster of the palmitoylated form of PSD-95 tagged using for the first time a genetically encoded antibody sensitive to palmitoylated form of PSD95 and imaged by STED microscopy. Nair et al. also investigated the organization of PSD-95 fused to mEOS by PALM and found $~ 150$-nm clusters. While the presence of PSD-95 cluster is observed by the three groups, the number of clusters is still controversial since MacGillavry et al. observed one cluster per PSD $(<10 \%$ of PSDs contain more than one PSD-95 cluster), whereas Fukata et al. and Nair et al. observed between one to four cluster per PSD depending of the PSD size ( $40 \%$ of PSDs contain more than one PSD-95 cluster). Recently, Blanpied's group reported an average of two nanoclusters of endogenous PSD-95 per synapse. ${ }^{129}$ In brain slices, these PSD95 subclusters have been recently reported as well, and both Broadhead et al. and Tang et al. found that $20 \%$ to $40 \%$ of PSDs contain more than one PSD-95 nanocluster, on PSD95 mEOS or GFP knock-in mice or endogenous PSD 95, respectively. ${ }^{104,129}$

Due to the large number of laboratories that have reported the postsynaptic nano-organization of PSD95 and AMPAR, this new concept discovered 3 years ago is now being currently accepted. One important question regarding this synaptic organization has been answered recently by the work of Blanpied's Lab, demonstrating the presence of presynapticpostsynaptic nanocolumns. ${ }^{129}$

It is optically challenging to realize multiple color experiments at the nanoscale because of drift during acquisition, or achromatisms, and so on. The solution they used was to couple a new cluster detection method based on tessellation ${ }^{130}$ and cross-correlation analysis to determine if two proteins are organized better than random. Tang et al. applied this analysis type on dual 3-D-dSTORM images to observe presynaptic scaffolding proteins as regulating synaptic membrane exocytosi (RIM) $1 / 2$ and the main postsynaptic scaffolding protein, PSD-95. RIM is known to play an important role in synaptic-vesicle docking through its interaction with MUNC13, which recruits calciumchannels. ${ }^{131-133}$ Tang et al. observed that RIM1/2 presents a clustered organization identical to PSD95 nanoclusters in both 
size and number of clusters. On the contrary, MUNC13 is more broadly distributed, and Bassoon seems randomly organized. ${ }^{129}$

Tang et al. ${ }^{129}$ demonstrated that presynaptic clusters of RIM1/2 are mainly aligned in front of postsynaptic clusters of PSD95. This study provides evidence for the existence of transsynaptic nanocolumns which coorganize the presynaptic machinery for glutamate release with the postsynaptic AMPAR nanodomains. This new concept reveals a molecular level of organization between pre- and postsynapses unexpected 20 years ago, which likely notably improves the efficiency of synaptic transmission. The molecular component responsible for this presynaptic-postsynaptic alignment remains to be identified. Deciphering the parameters that determine their regulation during physiological processes as maturation and plasticities will be important. Multiple candidates have been identified, such as neurexin/neuroligin, N-cadherin, leucine rich repeat transmembrane, or synCAM, but the relevant molecules are still unknown. ${ }^{134-137}$

The physiological impact of such an organization of the postsynaptic compartment on synaptic transmission properties was then investigated by using modeling. MacGillavry et al. used Monte Carlo simulation to determine the effect of the localization of glutamate release on uniform or clustered distribution of AMPARs and showed that the release of glutamate on AMPARs cluster increases the amplitude of mEPSCs compared to an "off cluster" release or release on a "uniform" distribution. ${ }^{64}$ Based on the same model, Nair et al. ${ }^{65}$ determined the impact not only of AMPAR density inside clusters, but also of the intercluster distance and cluster to release site distance on synaptic responses. Monte Carlo simulations suggested that all these parameters strongly impact the amplitude of mEPSCs. The density of AMPARs was the most sensitive parameter. On the contrary, a certain tolerance of a couple of tens of nanometer with respect to mEPSC amplitude was observed with respect to the location of the glutamate release site. Indeed, mEPSCs amplitude decreased only when the release site was at least $100 \mathrm{~nm}$ away from the nanodomain center.

In spines containing more than one AMPAR nanodomain, the average intercluster distance was measured of $450 \mathrm{~nm}$, with only $20 \%$ of clusters closer than $250 \mathrm{~nm}$ from one another. Monte Carlo simulations showed that when glutamate was released on top of a nanodomain, the second nanodomain is not activated if the intercluster distance is larger than $300 \mathrm{~nm}$, revealing a certain independence of each nanodomain. ${ }^{65}$

Experimentally, Nair et al. partly destabilized nanodomains to investigate the experimental importance of such an organization on synaptic properties. PSD-95 is one of the main organizers of AMPAR at synapses and two color super-resolution imaging of PSD-95 and AMPAR suggests a colocalization of both proteins. Knocking-down PSD-95 led to a $21 \%$ decrease of AMPAR number per nanodomain, which was correlated with a $20 \%$ decrease in mEPSCs amplitude. This correlation between nanodomain content in AMPAR and the amplitude of synaptic transmission suggests that AMPAR nanodomains could be responsible for the postsynaptic quantum of synaptic response.

This discovery of AMPAR nano-organization coupled to the concept of lateral diffusion changes our vision of the synaptic organization and function, but raises multiple questions. The previously reported studies present a new vision of the synapse at its stable state, but synapses are plastic organelles, able to adapt both to short- and long-term stimulation. Hence, one can postulate that modifications of AMPAR nanoscale organization could underlie various forms of synaptic plasticity. Many studies have brought indications of the molecular rearrangements taking place during plasticity at the whole synapse-diffraction limited-level; we now need to fuse these studies with the concept of lateral diffusion and nanoclustering of AMPAR to deliver a new vision of synaptic transmission regulation during plastic events.

\section{Activity Regulates the Dynamic Nano-Organization of AMPARs}

\subsection{Importance of the Dynamic Nano-Organization of AMPARs for Short-Term Plasticity}

Neurons are able to adapt their synaptic response at high frequency as a function of the previously received stimuli. Indeed, the amplitude of a second response is highly dependent on the delay that separates it from the first one. This mechanism, called short-term plasticity, has been abundantly described because it varies as a function of the type of neuron, the maturation status of the synapses, and so on and determines the capacity of the neuron to integrate and either filter or amplify the received signal. ${ }^{138}$ Until recently, regulation of paired pulse responses has solely been attributed to presynaptic modifications of transmitter release or AMPAR desensitization. Presynaptic short-term plasticity mechanisms largely involve variations in presynaptic calcium buffering capacities or availability of transmitter filled vesicles for release. If release probability is boosted by the first stimulus, this leads to paired pulse facilitation, whereas if release probability decreases, it leads to paired pulse depression. Postsynaptic AMPAR desensitization also participates in paired pulse depression at synapses with high release probability. ${ }^{139-141}$ However, it has been generally thought that at most synapses, and in particular at the Schaffer collateral-CA1 cell synapses, AMPAR desensitization does not participate in short-term plasticity. ${ }^{142}$ Generally, the impact of AMPAR desensitization on paired pulse synaptic responses is observed to be surprisingly lower than expected with respect to the AMPAR biophysical properties observed in heterologous systems. $^{143}$

The introduction of the concept of AMPAR lateral mobility in 2002 brought a new potentially important parameter. ${ }^{47}$ Indeed, the speed of the mobile receptors, around 0.1 to $1 \mu \mathrm{m}^{2} \mathrm{~s}^{-1}$, is compatible with the temporality of paired pulse synaptic events. In 2008, a role for AMPAR lateral mobility in tuning the rate of recovery from paired pulse depression was proposed. Heine et al. showed that the blockade of AMPAR lateral mobility through antibody crosslinking largely decreases the amplitude of the second synaptic response, promoting paired-pulse depression. ${ }^{77}$ The general idea underlying this study was that as AMPAR constantly diffuse inside synapses, their speed allows them to cross the PSD within tens of milliseconds. Thus, during a paired pulse response with an interstimulus interval in the tens of ms range, a significant amount of AMPAR can be spatially exchanged. After a first glutamate release, all receptors, and so among them the desensitized one, could thus be replaced by naïve receptors from adjacent regions. This could allow a faster recovery from synaptic depression. The conclusion of this work was that AMPAR lateral mobility could contribute to improve the synaptic response to high-frequency stimulation. 

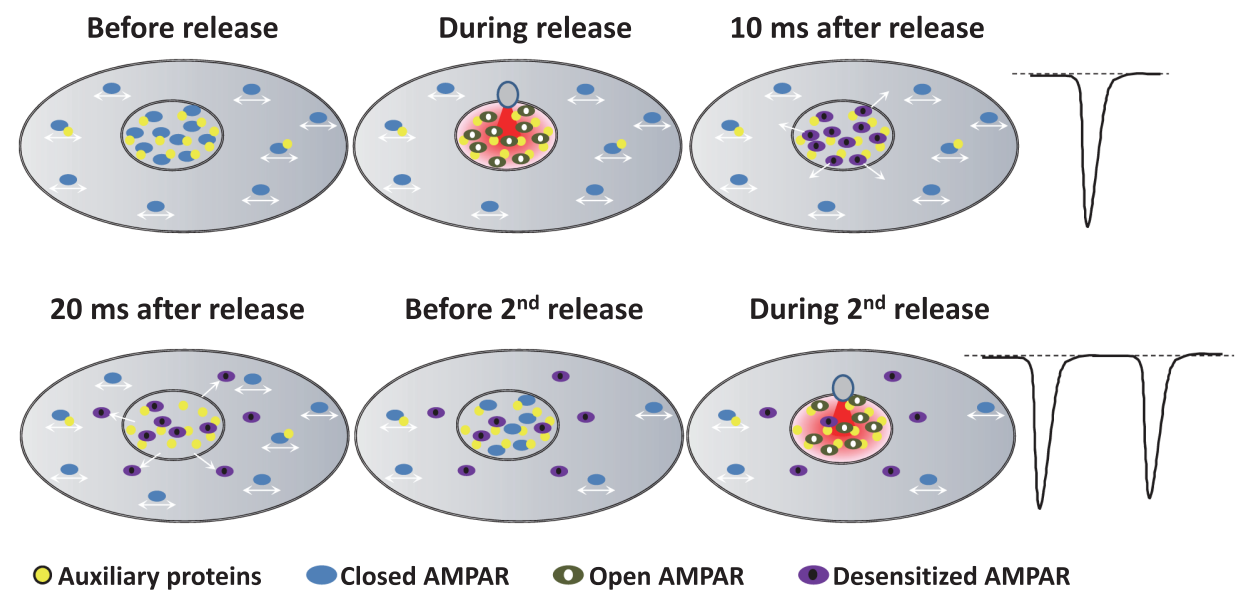

Fig. 4 Chronological scheme of the role of AMPAR lateral mobility on short-term paired pulse response. Before release, all receptors are closed, part of them are trapped inside clusters, the other diffusing freely. Just after release, if release happens on clusters, receptors in an area of $150 \mathrm{~nm}$ around the release site get opened and then rapidly desensitized, leading to classical synaptic currents. Rapidly, part of desensitized receptors unbind from their auxiliary proteins and diffuse out of the release site. Diffusive closed receptors can be trapped by the free auxiliary proteins, renewing the naïve AMPAR content inside the cluster. When a second release happens, part of receptors desensitized by the first release have diffused out of the area facing the release site, and most receptors under the release are in a closed state, available for activation by the second release. This conformational-dependent lateral mobility favors a sustained synaptic response at high release frequency.

The role of AMPAR diffusion on paired pulse responses could be even stronger if only desensitized receptors would diffuse out of the release site, whereas naïve receptors would replace them. Several studies reported that glutamate tends to increase AMPAR mobility, ${ }^{49,144}$ without clearly identifying the underlying molecular mechanism. Using conformational mutants and drug applications, Constals et al. demonstrated that desensitized receptors are more diffusive than opened or closed receptors. ${ }^{75}$ Glutamate induced unbinding, or at least uncoupling, between AMPARs and its main auxiliary protein stargazin has been described since $2004 .{ }^{145,146}$ The use of genetic fusion between AMPAR and stargazin and biochemical experiments confirmed that the glutamate-dependent mobility increase was due to a loss of affinity of desensitized receptors for their auxiliary proteins. ${ }^{75}$ This loss of AMPAR-TARP interaction is important for the recovery observed during pairedpulse depression experiments ${ }^{75}$ (Fig. 4). Other auxiliary proteins may also play a role in the recovery from depression, such as Shisa6, which traps AMPAR into synapses and prevents desensitization during synaptic activity. ${ }^{91}$

A model emerged from these studies, in which AMPARs are immobilized inside nanodomains by interacting with auxiliary proteins and scaffolding proteins. The first release of glutamate activates AMPAR, which then quickly desensitize. The associated conformational changes trigger an increase in AMPAR mobility, freeing them from TARP induced immobilization. The freely diffusive closed receptors can be specifically trapped at these free trapping sites, allowing a renewing of AMPAR inside the nanocluster in the tens of milliseconds. This specific glutamateinduced mobility of desensitized AMPAR can be at the root of the receptor turnover essential for fidelity of fast synaptic transmission. ${ }^{75}$ Such a model reconciles the role of AMPAR desensitization with their experimentally measured weak impact on paired pulse responses. A prediction of these results is that regulation of AMPAR mobility could adapt neuronal responses to bursting activity. It will be, therefore, of interest to determine the impact of AMPAR mobility on tuning network activity.

\subsection{Long-Term Plasticity}

While we described above how synapses can modify their shortterm responses, it has also been described half a century ago that they can regulate their responses on the long term. These mechanisms, called long-term plasticity, seem at least in part, to be at the basis of information storage and memory. ${ }^{18-20,34}$ It is now well established that these learning and memory mechanisms are mediated in large part by long lasting changes in the AMPAR mediated synaptic responses. The most thoroughly characterized examples of such synaptic plasticity are LTP and long-term depression (LTD). ${ }^{18,34}$

Since these first seminal papers, many laboratories worked to decipher the molecular mechanisms responsible for those events. It is now clear that LTP and LTD require the exocytosis and the endocytosis of AMPARs, respectively. These mechanisms trigger a regulation of the total amount of AMPAR at the cell surface. However, we previously described that the postsynapse is dynamically nano-organized and that both the dynamic and the organization of AMPAR regulate synaptic transmission properties. Recently, Monte Carlo-based simulation described the multiple molecular parameters that could lead to a potentiation. ${ }^{63}$ Those simulations revealed that an increase in AMPAR clustering inside nanodomains, or an increase in the number of AMPAR per nanodomain, or an improvement of the alignment between presynaptic release sites and AMPAR clusters, could trigger an increase in AMPAR response amplitude. Surprisingly, these models suggested that a $50 \%$ potentiation in synaptic current necessitates either a $100 \%$ to $200 \%$ increase in AMPAR number at the synapses, or only a modest increase in the AMPARs density into nanodomains. ${ }^{63,65}$ Based on those simulations and the discovery of the trapping of AMPAR into nanodomains, it is possible to postulate that 
LTP could be due to an increase in the density of AMPAR and/or an increase of the nanodomain size, or an improvement in the alignment between the presynaptic glutamate release site and the postsynaptic nanodomain. The use of super-resolution microscopy being quite recent in the field, this hypothesis has not been yet investigated, but previous studies could help us to support or invalidate some of these hypotheses.

First, regarding the hypothesis of an increase in AMPAR density inside nanodomains during LTP, d-STORM experiments allowed to determine that 20 to 25 receptors are contained inside a nanodomain. ${ }^{65,106,130}$ Structural properties of AMPARs indicate that an individual homomeric GluA2 AMPAR has a width of around $15 \mathrm{~nm}$, at its N-terminal domain. ${ }^{87}$ Even if some other studies determined that heteromeric GluA2/GluA3 AMPARs have a more compact NTD in an "O-shape,"147 an estimation of around $15 \mathrm{~nm}$ taking into account the presence of the various auxiliary proteins should be close to the reality, leading to an estimated area of $0.0002 \mu \mathrm{m}^{2}$ per receptor. ${ }^{148,149}$ The surface of a nanodomain is around $0.008 \mu \mathrm{m}^{2}$, corresponding to a diameter of $100 \mathrm{~nm} .{ }^{65}$ Based on mathematical compacting optimization calculation, a maximum of 35 receptors can be contained inside a single nanodomain. ${ }^{150}$ Considering the molecular arrangement inside the membrane as a nearly optimal organization, justified by the ability of AMPAR to exchange inside the nanodomain, we can conclude that the packing level of AMPAR is already likely close to its maximum at the basal state, making unlikely the hypothesis that an increase of AMPAR density inside nanodomains could underlie LTP.

Another hypothesis proposed to explain LTP is an improvement of the alignment of the presynaptic release site with AMPAR nanodomains. Modeling has demonstrated that such changes in preorganization-postorganization should improve both amplitude and reliability of synaptic transmission. 56,59,63 Tang et al. have investigated the effect of chemical-LTP on the transsynaptic alignment between RIM1/2 and PSD-95 clusters. They reported that nanocolumns are conserved after LTP induction, with an enrichment of PSD-95 clusters. Unfortunately they did not precisely quantify the potential nanoscale changes between glutamate release sites and AMPARs nanodomains alignment during LTP. ${ }^{129}$

The last hypothesis relates to the incorporation of new AMPAR during LTP. An increase in the total amount of surface AMPARs due to exocytosis as well as an immobilization at synaptic sites of surface receptors has been regularly observed after LTP induction. . $^{30,96,151,152}$ The use of single-molecule tracking allowed to investigate the molecular mechanisms responsible for the activity-dependent trapping of AMPAR inside the synapse. ${ }^{47,78,96}$ After N-méthyl-D-aspartic acid receptor (NMDAR) activation by a LTP protocol, the resulting calcium influx triggers CaMKII translocation from a dendritic position to the synapses, where it phosphorylates the CTerminal domain of various AMPAR subunits and auxiliary proteins. In the case of the AMPAR auxiliary protein stargazin, phosphorylation of the stretch of serines upstream of the c-terminal PDZ-binding domain changes the positive charges of the $\mathrm{C}$-tail to highly negative, inducing its repulsion from the negatively charged membrane lipids. This allows the unfolding of the C-tail and favors its interaction with the scaffolding proteins PSD95. ${ }^{92,93,96}$ Such a mechanism triggers a net increase in the synaptic trapping of AMPARs. However, whether AMPARs become trapped on pre-existing nanodomains or if new ones are created remains to be determined.
Other auxiliary proteins than stargazin could be implicated in this process. For example, gamma- 8 is required for LTP. ${ }^{100}$ All of those results strongly support the hypothesis that new synaptic immobilization slots for AMPAR are created during LTP induction. ${ }^{153}$ The discovery of the nanodomain organization of AMPAR inside synapses underlines the importance of the localization of such trapping events. Nanodomains have been identified as the place where AMPARs are immobilized. Thus, an increase in AMPAR trapping should be mediated by an increase either in the number of clusters, or in their sizes. Use of super-resolution microscopy should help to answer this question and provide further evidence of the highly dynamic reorganization of AMPARs at the nanoscale during LTP.

\section{Conclusion}

Application of super-resolution techniques in both live and fixed neurons has revealed a new and unexpected level of AMPAR organization inside synapses, allowing to tune our model of synaptic transmission. Indeed, single-particle tracking microscopy has demonstrated that lateral mobility of AMPAR impacts fast synaptic transmission by creating a constant turnover between desensitized and naïve receptors. Fixed and live super-resolution techniques led to the discovery of AMPAR nano-organization and led to the introduction of the notion of a postsynaptic quantum of response.

Even if the interplay between long-term plasticity and AMPAR nanoscale organization has not yet been determined, previous work tends to support the notion that an increase in molecular trapping into nanodomains during LTP is at least one cause of the increase in synaptic response.

One century after the first description of the synapse, our vision largely evolved, due to technical improvements. A modern synapse is not a homogenously organized organelle but a complex assembly of nanoscale compartments whose individual components exchange constantly. This level of organization seems adapted to optimize the efficiency of use of the presynaptically released glutamate. Indeed, if as it as been recently shown, presynaptic release sites are aligned with AMPAR nanoclusters, the various glutamate receptors will be organized at a distance from release site relative to their affinity for glutamate. ${ }^{129}$ The higher their affinity (as for NMDAR or mGluR) the less stringent the location of receptors with respect to the release site.

Regulation of AMPAR localization and trafficking heavily relies on a complex interplay between the AMPAR complex composition and the level of phosphorylation of the various cytoplasmic tails of the complex-be it receptors or their auxiliary proteins. The next step will be the understanding of the role of each auxiliary protein on AMPAR nanoscale organization and the impact on synaptic transmission properties during the various state of the synapse, during development and plasticity events, and in the different brain regions.

\section{Acknowledgments}

We express our thanks to Corey Butler for discussions and corrections of the manuscript. This work was supported by the ANR NanoDom, Labex BRAIN, and ANR-10-INBS-04 FranceBioImaging, Centre National de la Recherche Scientifique, ERC Grant ADOS 339541 to D.C. and a fellowship from the MESR to B.C. The authors have no relevant financial interests in this paper and no other potential conflicts of interest to disclose. 


\section{References}

1. S. R. Y. Cajal, "Estudios sobre el plan estructural y composición histológica de los centros nerviosos adicionados de consideraciones fisiológicas fundadas en los nuevos descubrimientos," in Textura del Sistema Nervioso del Hombre y de los Vertebrados, Imprenta y Librería de Nicolás Moya, Madrid (1904).

2. E. D. De Robertis and H. S. Bennett, "Some features of the submicroscopic morphology of synapses in frog and earthworm," J. Biophys. Biochem. Cytol. 1(1), 47-58 (1955).

3. S. L. Palay and G. E. Palade, "The fine structure of neurons," J. Biophys. Biochem. Cytol. 1(1), 69-88 (1955).

4. E. D. De Robertis and H. S. Bennett, "A submicroscopic vesicular component of Schwann cells and nerve satellite cells," Exp. Cell Res. 6(2), 543-545 (1954).

5. S. L. Palay, "Synapses in the central nervous system," J. Biophys. Biochem. Cytol. 2(4 Suppl), 193-202 (1956).

6. J. Del Castillo and B. Katz, "Quantal components of the end-plate potential," J. Physiol. 124(3), 560-573 (1954).

7. P. Fatt and B. Katz, "An analysis of the end-plate potential recorded with an intracellular electrode," J. Physiol. 115(3), 320-370 (1951).

8. O. P. Hamill et al., "Improved patch-clamp techniques for highresolution current recording from cells and cell-free membrane patches," Pflugers Arch. Eur. J. Physiol. 391(2), 85-100 (1981).

9. M. Hollmann and S. Heinemann, "Cloned glutamate receptors," Annu. Rev. Neurosci. 17, 31-108 (1994).

10. K. O. Cho, C. A. Hunt, and M. B. Kennedy, "The rat brain postsynaptic density fraction contains a homolog of the Drosophila discs-large tumor suppressor protein," Neuron 9(5), 929-942 (1992).

11. C. A. Hunt, L. J. Schenker, and M. B. Kennedy, "PSD-95 is associated with the postsynaptic density and not with the presynaptic membrane at forebrain synapses," J. Neurosci. 16(4), 1380-1388 (1996).

12. H. C. Kornau et al., "Domain interaction between NMDA receptor subunits and the postsynaptic density protein PSD-95," Science 269(5231), 1737-1740 (1995).

13. S. G. Grant, "SnapShot: organizational principles of the postsynaptic proteome," Neuron 80(2), 534.e1 (2013).

14. H. Husi and S. G. Grant, "Proteomics of the nervous system," Trends Neurosci. 24(5), 259-266 (2001).

15. K. W. Li et al., "Proteomics analysis of rat brain postsynaptic density. Implications of the diverse protein functional groups for the integration of synaptic physiology," J. Biol. Chem. 279(2), 987-1002 (2004).

16. R. S. Walikonis et al., "Identification of proteins in the postsynaptic density fraction by mass spectrometry," J. Neurosci. 20(11), 40694080 (2000).

17. T. V. Bliss and T. Lomo, "Long-lasting potentiation of synaptic transmission in the dentate area of the anaesthetized rabbit following stimulation of the perforant path," J. Physiol. 232(2), 331-356 (1973).

18. S. M. Dudek and M. F. Bear, "Homosynaptic long-term depression in area CA1 of hippocampus and effects of N-methyl-D-aspartate receptor blockade," Proc. Natl. Acad. Sci. U. S. A. 89(10), 4363-4367 (1992).

19. E. R. Kandel, "Genes, synapses, and long-term memory," J. Cell. Physiol. 173(2), 124-125 (1997).

20. R. E. Nicholls et al., "Transgenic mice lacking NMDAR-dependent LTD exhibit deficits in behavioral flexibility," Neuron 58(1), 104-117 (2008).

21. S. Brenowitz and L. O. Trussell, "Minimizing synaptic depression by control of release probability," J. Neurosci. 21(6), 1857-1867 (2001).

22. J. M. Bekkers and C. F. Stevens, "Presynaptic mechanism for longterm potentiation in the hippocampus," Nature 346(6286), 724-729 (1990).

23. H. Kamiya, S. Sawada, and C. Yamamoto, "Persistent enhancement of transmitter release accompanying long-term potentiation in the guinea pig hippocampus," Neurosci. Lett. 130(2), 259-262 (1991).

24. R. Malinow and R. W. Tsien, "Presynaptic enhancement shown by whole-cell recordings of long-term potentiation in hippocampal slices," Nature 346(6280), 177-180 (1990).

25. S. N. Davies et al., "Temporally distinct pre- and post-synaptic mechanisms maintain long-term potentiation," Nature 338(6215), 500-503 (1989).

26. J. A. Kauer, R. C. Malenka, and R. A. Nicoll, "A persistent postsynaptic modification mediates long-term potentiation in the hippocampus," Neuron 1(10), 911-917 (1988).
27. R. C. Malenka et al., "Postsynaptic calcium is sufficient for potentiation of hippocampal synaptic transmission," Science 242(4875), 81-84 (1988).

28. D. J. Perkel et al., "The role of $\mathrm{Ca}^{2+}$ entry via synaptically activated NMDA receptors in the induction of long-term potentiation," Neuron 11(5), 817-823 (1993).

29. S. Choi, J. Klingauf, and R. W. Tsien, "Postfusional regulation of cleft glutamate concentration during LTP at 'silent synapses'," Nat. Neurosci. 3(4), 330-336 (2000).

30. J. T. Isaac, R. A. Nicoll, and R. C. Malenka, "Evidence for silent synapses: implications for the expression of LTP," Neuron 15(2), 427-434 (1995).

31. D. Liao, N. A. Hessler, and R. Malinow, "Activation of postsynaptically silent synapses during pairing-induced LTP in CA1 region of hippocampal slice," Nature 375(6530), 400-404 (1995).

32. G. Carlson, Y. Wang, and B. E. Alger, "Endocannabinoids facilitate the induction of LTP in the hippocampus," Nat. Neurosci. 5(8), 723-724 (2002).

33. J. P. Terranova et al., "Inhibition of long-term potentiation in rat hippocampal slices by anandamide and WIN55212-2: reversal by SR141716 A, a selective antagonist of CB1 cannabinoid receptors," Naunyn-Schmiedeberg's Arch. Pharmacol. 352(5), 576-579 (1995).

34. T. V. Bliss and A. R. Gardner-Medwin, "Long-lasting potentiation of synaptic transmission in the dentate area of the unanaestetized rabbit following stimulation of the perforant path," J. Physiol. 232(2), 357-374 (1973).

35. G. Lynch and M. Baudry, "The biochemistry of memory: a new and specific hypothesis," Science 224(4653), 1057-1063 (1984).

36. G. Lynch et al., "Intracellular injections of EGTA block induction of hippocampal long-term potentiation," Nature 305(5936), 719-721 (1983).

37. T. V. Bliss and G. L. Collingridge, "A synaptic model of memory: long-term potentiation in the hippocampus," Nature 361(6407), 31-39 (1993).

38. G. Lynch, S. Halpain, and M. Baudry, "Effects of high-frequency synaptic stimulation on glumate receptor binding studied with a modified in vitro hippocampal slice preparation," Brain Res. 244(1), 101-111 (1982).

39. D. Muller and G. Lynch, "Long-term potentiation differentially affects two components of synaptic responses in hippocampus," Proc. Natl. Acad. Sci. U. S. A. 85(23), 9346-9350 (1988).

40. E. C. Beattie et al., "Regulation of AMPA receptor endocytosis by a signaling mechanism shared with LTD," Nat. Neurosci. 3(12), 1291-1300 (2000).

41. W. Lu et al., "Activation of synaptic NMDA receptors induces membrane insertion of new AMPA receptors and LTP in cultured hippocampal neurons," Neuron 29(1), 243-254 (2001).

42. D. S. Bredt and R. A. Nicoll, "AMPA receptor trafficking at excitatory synapses," Neuron 40(2), 361-379 (2003).

43. R. C. Carroll et al., "Dynamin-dependent endocytosis of ionotropic glutamate receptors," Proc. Natl. Acad. Sci. U. S. A. 96(24), 14112-14117 (1999).

44. C. Luscher et al., "Role of AMPA receptor cycling in synaptic transmission and plasticity," Neuron 24(3), 649-658 (1999).

45. S. H. Shi et al., "Rapid spine delivery and redistribution of AMPA receptors after synaptic NMDA receptor activation," Science 284(5421), 1811-1816 (1999).

46. I. Song and R. L. Huganir, "Regulation of AMPA receptors during synaptic plasticity," Trends Neurosci. 25(11), 578-588 (2002).

47. A. J. Borgdorff and D. Choquet, "Regulation of AMPA receptor lateral movements," Nature 417(6889), 649-653 (2002).

48. D. Choquet and A. Triller, "The role of receptor diffusion in the organization of the postsynaptic membrane," Nat. Rev. 4(4), 251-265 (2003).

49. C. Tardin et al., "Direct imaging of lateral movements of AMPA receptors inside synapses," EMBO J. 22(18), 4656-4665 (2003).

50. A. Baude et al., "High-resolution immunogold localization of AMPA type glutamate receptor subunits at synaptic and non-synaptic sites in rat hippocampus," Neuroscience 69(4), 1031-1055 (1995).

51. M. Antal et al., "Numbers, densities, and colocalization of AMPA- and NMDA-type glutamate receptors at individual synapses in the superficial spinal dorsal horn of rats," J. Neurosci. 28(39), 9692-9701 (2008). 
52. M. Masugi-Tokita et al., "Number and density of AMPA receptors in individual synapses in the rat cerebellum as revealed by SDS-digested freeze-fracture replica labeling," J. Neurosci. 27(8), 2135-2144 (2007).

53. J. Tanaka et al., "Number and density of AMPA receptors in single synapses in immature cerebellum," J. Neurosci. 25(4), 799-807 (2005).

54. G. Liu, S. Choi, and R. W. Tsien, "Variability of neurotransmitter concentration and nonsaturation of postsynaptic AMPA receptors at synapses in hippocampal cultures and slices," Neuron 22(2), 395-409 (1999).

55. K. M. Franks, T. M. Bartol, and T. J. Sejnowski, "A Monte Carlo model reveals independent signaling at central glutamatergic synapses," Biophys. J. 83(5), 2333-2348 (2002).

56. K. M. Franks, C. F. Stevens, and T. J. Sejnowski, "Independent sources of quantal variability at single glutamatergic synapses," J. Neurosci. 23(8), 3186-3195 (2003).

57. T. A. Nielsen, D. A. DiGregorio, and R. A. Silver, "Modulation of glutamate mobility reveals the mechanism underlying slow-rising AMPAR EPSCs and the diffusion coefficient in the synaptic cleft," Neuron 42(5), 757-771 (2004).

58. L. P. Savtchenko, S. Sylantyev, and D. A. Rusakov, "Central synapses release a resource-efficient amount of glutamate," Nat. Neurosci. 16(1), 10-12 (2013).

59. E. Tarusawa et al., "Input-specific intrasynaptic arrangements of ionotropic glutamate receptors and their impact on postsynaptic responses," J. Neurosci. 29(41), 12896-12908 (2009).

60. J. D. Clements, "Transmitter timecourse in the synaptic cleft: its role in central synaptic function," Trends Neurosci. 19(5), 163-171 (1996).

61. J. D. Clements et al., "The time course of glutamate in the synaptic cleft," Science 258(5087), 1498-1501 (1992).

62. J. E. Lisman, S. Raghavachari, and R. W. Tsien, "The sequence of events that underlie quantal transmission at central glutamatergic synapses," Nat. Rev. 8(8), 597-609 (2007).

63. L. P. Savtchenko and D. A. Rusakov, "Moderate AMPA receptor clustering on the nanoscale can efficiently potentiate synaptic current," Philos. Trans. R. Soc. London, Ser. B 369(1633), 20130167 (2014).

64. H. D. MacGillavry et al., "Nanoscale scaffolding domains within the postsynaptic density concentrate synaptic AMPA receptors," Neuron 78(4), 615-622 (2013).

65. D. Nair et al., "Super-resolution imaging reveals that AMPA receptors inside synapses are dynamically organized in nanodomains regulated by PSD95," J. Neurosci. 33(32), 13204-13224 (2013).

66. S. J. Singer and G. L. Nicolson, "The fluid mosaic model of the structure of cell membranes," Science 175(4023), 720-731 (1972).

67. D. Axelrod et al., "Mobility measurement by analysis of fluorescence photobleaching recovery kinetics," Biophys. J. 16(9), 1055-1069 (1976).

68. D. Axelrod et al., "Lateral motion of fluorescently labeled acetylcholine receptors in membranes of developing muscle fibers," Proc. Natl. Acad. Sci. U. S. A. 73(12), 4594-4598 (1976).

69. N. M. Chao, S. H. Young, and M. M. Poo, "Localization of cell membrane components by surface diffusion into a "trap"," Biophys. J. 36(1), 139-153 (1981).

70. S. H. Young and M. M. Poo, "Rapid lateral diffusion of extrajunctional acetylcholine receptors in the developing muscle membrane of Xenopus tadpole," J. Neurosci. 3(1), 225-231 (1983).

71. J. Gelles, B. J. Schnapp, and M. P. Sheetz, "Tracking kinesin-driven movements with nanometre-scale precision," Nature 331(6155), 450 453 (1988).

72. A. Kusumi, Y. Sako, and M. Yamamoto, "Confined lateral diffusion of membrane receptors as studied by single particle tracking (nanovid microscopy). Effects of calcium-induced differentiation in cultured epithelial cells," Biophys. J. 65(5), 2021-2040 (1993).

73. K. Suzuki et al., "Rapid hop diffusion of a G-protein-coupled receptor in the plasma membrane as revealed by single-molecule techniques," Biophys. J. 88(5), 3659-3680 (2005).

74. J. Meier et al., "Fast and reversible trapping of surface glycine receptors by gephyrin," Nat. Neurosci. 4(3), 253-260 (2001).

75. A. Constals et al., "Glutamate-induced AMPA receptor desensitization increases their mobility and modulates short-term plasticity through unbinding from stargazin," Neuron 85(4), 787-803 (2015).

76. M. D. Ehlers et al., "Diffusional trapping of GluR1 AMPA receptors by input-specific synaptic activity," Neuron 54(3), 447-460 (2007).
77. M. Heine et al., "Surface mobility of postsynaptic AMPARs tunes synaptic transmission," Science 320(5873), 201-205 (2008).

78. E. M. Petrini et al., "Endocytic trafficking and recycling maintain a pool of mobile surface AMPA receptors required for synaptic potentiation," Neuron 63(1), 92-105 (2009).

79. P. Klemmer, A. B. Smit, and K. W. Li, "Proteomics analysis of immuno-precipitated synaptic protein complexes," J. Proteomics 72(1), 82-90 (2009).

80. J. Schwenk et al., "High-resolution proteomics unravel architecture and molecular diversity of native AMPA receptor complexes," Neuron 74(4), 621-633 (2012).

81. J. Schwenk et al., "Functional proteomics identify cornichon proteins as auxiliary subunits of AMPA receptors," Science 323(5919), 13131319 (2009).

82. J. von Engelhardt et al., "CKAMP44: a brain-specific protein attenuating short-term synaptic plasticity in the dentate gyrus," Science 327(5972), 1518-1522 (2010).

83. V. A. Letts et al., "The mouse stargazer gene encodes a neuronal $\mathrm{Ca}^{2+}$-channel gamma subunit," Nat. Genet. 19(4), 340-347 (1998).

84. L. Chen et al., "Stargazin regulates synaptic targeting of AMPA receptors by two distinct mechanisms," Nature 408(6815), 936-943 (2000).

85. J. Choi et al., "Phosphorylation of stargazin by protein kinase A regulates its interaction with PSD-95," J. Biol. Chem. 277(14), 12359-12363 (2002).

86. J. Schwenk et al., "Regional diversity and developmental dynamics of the AMPA-receptor proteome in the mammalian brain," Neuron $\mathbf{8 4}(1)$, 41-54 (2014).

87. A. I. Sobolevsky, M. P. Rosconi, and E. Gouaux, "X-ray structure, symmetry and mechanism of an AMPA-subtype glutamate receptor," Nature 462(7274), 745-756 (2009).

88. S. Tomita et al., "Functional studies and distribution define a family of transmembrane AMPA receptor regulatory proteins," J. Cell Biol. 161(4), 805-816 (2003).

89. M. B. Gill et al., "Cornichon-2 modulates AMPA receptor-transmembrane AMPA receptor regulatory protein assembly to dictate gating and pharmacology," J. Neurosci. 31(18), 6928-6938 (2011).

90. A. R. Karataeva et al., "C-terminal interactors of the AMPA receptor auxiliary subunit Shisa9," PLoS One 9(2), e87360 (2014).

91. R. V. Klaassen et al., "Shisa6 traps AMPA receptors at postsynaptic sites and prevents their desensitization during synaptic activity," Nat. Commun. 7, 10682 (2016).

92. S. Tomita et al., "Bidirectional synaptic plasticity regulated by phosphorylation of stargazin-like TARPs," Neuron 45(2), 269-277 (2005).

93. A. S. Hafner et al., "Lengthening of the stargazin cytoplasmic tail increases synaptic transmission by promoting interaction to deeper domains of PSD-95," Neuron 86(2), 475-489 (2015).

94. A. Sumioka, D. Yan, and S. Tomita, "TARP phosphorylation regulates synaptic AMPA receptors through lipid bilayers," Neuron 66(5), 755767 (2010).

95. C. Bats, L. Groc, and D. Choquet, "The interaction between Stargazin and PSD-95 regulates AMPA receptor surface trafficking," Neuron 53(5), 719-734 (2007).

96. P. Opazo et al., "CaMKII triggers the diffusional trapping of surface AMPARs through phosphorylation of stargazin," Neuron 67(2), 239252 (2010).

97. M. Sainlos et al., "Biomimetic divalent ligands for the acute disruption of synaptic AMPAR stabilization," Nat. Chem. Biol. 7(2), 81-91 (2010).

98. C. Bats et al., "Channel properties reveal differential expression of TARPed and TARPless AMPARs in stargazer neurons," Nat. Neurosci. 15(6), 853-861 (2012).

99. A. S. Kato et al., "New transmembrane AMPA receptor regulatory protein isoform, gamma-7, differentially regulates AMPA receptors," J. Neurosci. 27(18), 4969-4977 (2007).

100. N. Rouach et al., "TARP gamma-8 controls hippocampal AMPA receptor number, distribution and synaptic plasticity," Nat. Neurosci. 8(11), 1525-1533 (2005).

101. A. Sumioka et al., "PDZ binding of TARPgamma- 8 controls synaptic transmission but not synaptic plasticity," Nat. Neurosci. 14(11), 14101412 (2011).

102. A. S. Kato et al., "Hippocampal AMPA receptor gating controlled by both TARP and cornichon proteins," Neuron 68(6), 1082-1096 (2010). 
103. P. Farrow et al., "Auxiliary subunits of the CKAMP family differentially modulate AMPA receptor properties," eLife 4, e09693 (2015).

104. M. J. Broadhead et al., "PSD95 nanoclusters are postsynaptic building blocks in hippocampus circuits," Sci. Rep. 6, 24626 (2016).

105. Y. Fukata et al., "Local palmitoylation cycles define activity-regulated postsynaptic subdomains," J. Cell Biol. 202(1), 145-161 (2013).

106. E. Hosy, C. Butler, and J. B. Sibarita, "Organization and dynamics of AMPA receptors inside synapses-nano-organization of AMPA receptors and main synaptic scaffolding proteins revealed by superresolution imaging," Curr. Opin. Chem. Biol. 20, 120-126 (2014).

107. B. Albrecht et al., "Spatially modulated illumination microscopy: online visualization of intensity distribution and prediction of nanometer precision of axial distance measurements by computer simulations," J. Biomed. Opt. 6(3), 292-299 (2001).

108. E. Betzig, "Proposed method for molecular optical imaging," Opt. Lett. 20(3), 237-239 (1995).

109. E. Betzig et al., "Imaging intracellular fluorescent proteins at nanometer resolution," Science 313(5793), 1642-1645 (2006).

110. G. Giannone et al., "Dynamic superresolution imaging of endogenous proteins on living cells at ultra-high density," Biophys. J. 99(4), 13031310 (2010).

111. M. G. Gustafsson, "Surpassing the lateral resolution limit by a factor of two using structured illumination microscopy," J. Microsc. 198(Pt 2), 82-87 (2000).

112. M. Heilemann et al., "Subdiffraction-resolution fluorescence imaging with conventional fluorescent probes," Angew. Chem. Int. Ed. 47(33), 6172-6176 (2008).

113. S. W. Hell and J. Wichmann, "Breaking the diffraction resolution limit by stimulated emission: stimulated-emission-depletion fluorescence microscopy," Opt. Lett. 19(11), 780-782 (1994).

114. S. Manley et al., "High-density mapping of single-molecule trajectories with photoactivated localization microscopy," Nat. Methods 5(2), 155-157 (2008).

115. M. J. Rust, M. Bates, and X. Zhuang, "Sub-diffraction-limit imaging by stochastic optical reconstruction microscopy (STORM)," Nat. Methods 3(10), 793-796 (2006).

116. M. Bates et al., "Multicolor super-resolution imaging with photoswitchable fluorescent probes," Science 317(5845), 1749-1753 (2007).

117. B. Huang et al., "Whole-cell 3D STORM reveals interactions between cellular structures with nanometer-scale resolution," Nat. Methods 5(12), 1047-1052 (2008).

118. B. Huang et al., "Three-dimensional super-resolution imaging by stochastic optical reconstruction microscopy," Science 319(5864), 810-813 (2008).

119. M. F. Juette et al., "Three-dimensional sub-100 nm resolution fluorescence microscopy of thick samples," Nat. Methods 5(6), 527-529 (2008).

120. A. Vaziri et al., "Multilayer three-dimensional super resolution imaging of thick biological samples," Proc. Natl. Acad. Sci. U. S. A. 105(51), 20221-20226 (2008).

121. P. Bethge et al., "Two-photon excitation STED microscopy in two colors in acute brain slices," Biophys. J. 104(4), 778-785 (2013).

122. A. Dani et al., "Superresolution imaging of chemical synapses in the brain," Neuron 68(5), 843-856 (2010).

123. J. Tonnesen et al., "Two-color STED microscopy of living synapses using a single laser-beam pair," Biophys. J. 101(10), 2545-2552 (2011).

124. N. T. Urban et al., "STED nanoscopy of actin dynamics in synapses deep inside living brain slices," Biophys. J. 101(5), 1277-1284 (2011).

125. S. Berning et al., "Nanoscopy in a living mouse brain," Science 335(6068), 551-551 (2012).

126. B. C. Chen et al., "Lattice light-sheet microscopy: imaging molecules to embryos at high spatiotemporal resolution," Science 346(6208), 1257998 (2014).

127. R. Galland et al., "3D high- and super-resolution imaging using single-objective SPIM," Nat. Methods 12(7), 641-644 (2015).

128. L. Gao et al., "Noninvasive imaging beyond the diffraction limit of 3D dynamics in thickly fluorescent specimens," Cell 151(6), 1370-1385 (2012).

129. A. H. Tang et al., "A trans-synaptic nanocolumn aligns neurotransmitter release to receptors," Nature 536(7615), 210-214 (2016).
130. F. Levet et al., "SR-Tesseler: a method to segment and quantify localization-based super-resolution microscopy data," Nat. Methods 12(11), 1065-1071 (2015).

131. L. Deng et al., "RIM proteins activate vesicle priming by reversing autoinhibitory homodimerization of Munc13," Neuron 69(2), 317-331 (2011).

132. Y. Han et al., "RIM determines $\mathrm{Ca}^{(2)+}$ channel density and vesicle docking at the presynaptic active zone," Neuron 69(2), 304-316 (2011).

133. K. S. Liu et al., "RIM-binding protein, a central part of the active zone, is essential for neurotransmitter release," Science 334(6062), 15651569 (2011).

134. I. Chamma et al., "Mapping the dynamics and nanoscale organization of synaptic adhesion proteins using monomeric streptavidin," Nat. Commun. 7, 10773 (2016).

135. K. Perez de Arce et al., "Topographic mapping of the synaptic cleft into adhesive nanodomains," Neuron 88(6), 1165-1172 (2015).

136. L. Saglietti et al., "Extracellular interactions between GluR2 and N-cadherin in spine regulation," Neuron 54(3), 461-477 (2007).

137. P. Scheiffele et al., "Neuroligin expressed in nonneuronal cells triggers presynaptic development in contacting axons," Cell 101(6), 657-669 (2000).

138. R. S. Zucker and W. G. Regehr, "Short-term synaptic plasticity," Annu. Rev. Physiol. 64, 355-405 (2002).

139. D. A. DiGregorio et al., "Desensitization properties of AMPA receptors at the cerebellar mossy fiber granule cell synapse," J. Neurosci. 27(31), 8344-8357 (2007).

140. M. Koike-Tani et al., "Involvement of AMPA receptor desensitization in short-term synaptic depression at the calyx of Held in developing rats," J. Physiol. 586(9), 2263-2275 (2008).

141. A. Rozov and N. Burnashev, "Polyamine-dependent facilitation of postsynaptic AMPA receptors counteracts paired-pulse depression," Nature 401(6753), 594-598 (1999).

142. G. O. Hjelmstad et al., "Lack of AMPA receptor desensitization during basal synaptic transmission in the hippocampal slice," J. Neurophysiol. 81(6), 3096-3099 (1999).

143. C. Auger and A. Marty, "Quantal currents at single-site central synapses," J. Physiol. 526 (Pt 1), 3-11 (2000).

144. A. G. Petzoldt et al., "Gating characteristics control glutamate receptor distribution and trafficking in vivo," Curr. Biol. 24(17), 2059-2065 (2014).

145. M. Morimoto-Tomita et al., "Autoinactivation of neuronal AMPA receptors via glutamate-regulated TARP interaction," Neuron 61(1), 101-112 (2009).

146. S. Tomita et al., "Dynamic interaction of stargazin-like TARPs with cycling AMPA receptors at synapses," Science 303(5663), 1508-1511 (2004).

147. B. Herguedas et al., "Structure and organization of heteromeric AMPA-type glutamate receptors," Science 352(6285), aad3873 (2016).

148. N. Armstrong et al., "Measurement of conformational changes accompanying desensitization in an ionotropic glutamate receptor," Cell 127(1), 85-97 (2006).

149. J. Krieger, I. Bahar, and I. H. Greger, "Structure, dynamics, and allosteric potential of ionotropic glutamate receptor N-terminal domains," Biophys. J. 109(6), 1136-1148 (2015).

150. R. L. Graham et al., "Dense packings of congruent circles in a circle," Discrete Math. 181, 139-154 (1998).

151. M. Park et al., "Recycling endosomes supply AMPA receptors for LTP," Science 305(5692), 1972-1975 (2004).

152. Z. Szepesi et al., "Synaptically released matrix metalloproteinase activity in control of structural plasticity and the cell surface distribution of GluA1-AMPA receptors," PLoS One 9(5), e98274 (2014).

153. P. Opazo and D. Choquet, "A three-step model for the synaptic recruitment of AMPA receptors," Mol. Cell. Neurosci. 46(1), 1-8 (2011).

Benjamin Compans received his master's degree in neuroscience from the University of Bordeaux. He is a PhD student at the Interdisciplinary Institute for Neuroscience, Bordeaux, France, in Dr. Daniel Choquet's lab. During his PhD under the supervision of Dr. Eric Hosy, he aims to understand how the dynamic nanoorganization of AMPA receptors contributes to the establishment of synaptic plasticity. 
Daniel Choquet received his engineering degree from Ecole Centrale, Paris, France, and his PhD with Henri Korn from Pasteur Institute, Paris. He was a CNRS research officer in 1988 and a postdoc with Michael Sheetz at Duke University, North Carolina, USA. Currently, he is a director of the Institute for Interdisciplinary Neuroscience and the Bordeaux Imaging Center, Bordeaux, France, and a director of the BRAIN excellence cluster. He runs an interdisciplinary program on the use of high-resolution imaging to study the trafficking of neurotransmitter receptors in neurons.
Eric Hosy received his $\mathrm{PhD}$ in plant field in Montpellier, then studied channel structure function on KATP channel in Grenoble. He started working in neuroscience in 2006 in Rotterdam before moving to Daniel Choquet's Group, where he has been recruited at the CNRS in 2009. He is the coinventor of the U-PAINT technique and develops thematic centered around the coupling of live super-resolution technique and electrophysiology to determine the physiological role of AMPA receptor nano-organization. 